\section{Reduzierte TH1-Reaktion bei Schizophrenie}

In der Pathogenese der Schizophrenie spielen möglicherweise immunologische Prozesse eine Rolle. Manche Forscher vermuten, dass es bei den Patienten zu einer Abschwächung der zellulären Immunreaktion kommt. Lässt sich diese Hypothese klinisch untermauern?

$M$ ehrere immunologische Studien bei schizophrenen Patienten weisen auf eine verminderte Aktivität der zellulären Immunabwehr bei der Erkrankung hin, während die humorale Reaktion überaktiv zu sein scheint. In-vitroUntersuchungen zeigen eine Reduktion TH1-induzierter Zytokine wie Interferon- $\gamma$ oder Interleukin- 2 .

Um einer möglichen Aktivitätsminderung der TH1-Zellen bei Schizophrenie auf die Spur zu kommen, untersuchten Münchner Psychiater bei Erkrankten die Typ-IV-Immunreaktion, bei der TH1-Helferzellen eine wichtige Rolle spielen. Sie unterzogen 30 Patienten mit diagnostizierter Schizophrenie und 30 gesunde Probanden - jeweils ohne Hautallergien - einem standardi- sierten intradermalen Stempeltest. Dieser Test (Multitest Immignost ${ }^{\circledR}$ ) erfasst speziell Typ-IV-Reaktionen gegen Antigene, mit denen das humane Immunsystem

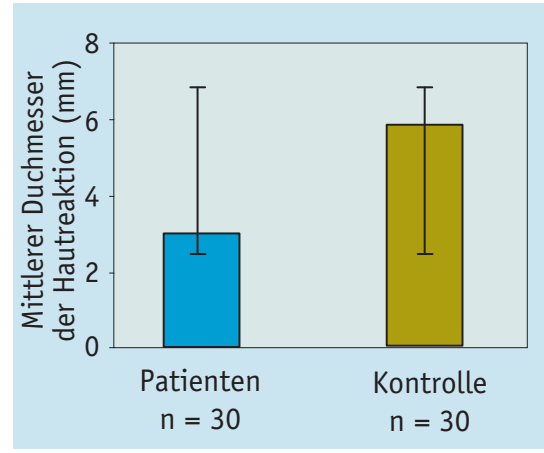

Hautreaktivität vom Typ IV bei Patienten mit Schizophrenie bereits in Kontakt gekommen ist, wie Impfstoffe oder häufige Erreger.

Bei den Patienten mit Schizophrenie zeigten sich insgesamt signifikant seltener positive Hautreaktionen als bei den Kontrollpersonen (24 vs. 51). Gegen Tetanus- und Diphtherie-Antigene reagierten die schizophrenen Patienten ebenfalls signifikant seltener positiv. Für die anderen untersuchten Antigene waren nur in der Tendenz seltener Reaktionen nachweisbar. Das Ausmaß der positiven Hautreaktionen war bei den erkrankten Patienten signifikant gerimger als bei den gesunden Probanden.

Fazit: Patienten mit Schizophrenie reagieren auf einen Typ-IV-anhängigen Hauttest seltener und schwächer als gesunde Kontrollpersonen. Dies unterstreicht die Hypothese, dass bei Schizophrenie eine Abschwächung der TH1vermittelten zellulären Immunantwort vorliegen könnte.

Riedel $\mathbf{M}$ et al. Decreased T cellular immune response in schizophrenic patients. J Psychiatr Res 2007; 41: 3-7

\title{
Multiple Chemical Sensitivity - nur ein Schlagwort?
}

\section{Das Phänomen der Multiple Chemical Sensitivity (MCS) wird nach wie vor kontrovers diskutiert. Handelt es sich um eine echte orga- nische Erkrankung oder um eine psychosomatische Reaktion? Eine Metaanalyse sollte jetzt Licht ins Dunkel bringen.}

U nter MCS versteht man eine Reihe von körperlichen Symptomen wie beispielsweise Übelkeit, Muskelschmerzen, Erschöpfungszustände oder Konzentrationsstörungen, die bei Exposition gegenüber niedrig konzentrierten chemischen Stoffen auftreten. Als auslösende Chemikalien werden vorwiegend Benzin, Parfums, Pestizide oder auch Druckfarben genannt. Manche Forscher sehen MCS als eine Reaktion unterschiedlicher Organsysteme. Andere vermuten eine individuell erlernte psychosomatische Reaktion auf Gerüche.

Psychiater vom King's College in London unterzogen Studien zu MCS ei- ner Metaanalyse. An diesen Untersuchungen waren insgesamt 784 Personen beteiligt gewesen, die angegeben hatten, an MCS zu leiden. Es waren in allen Studien standardisierte Provokationstests vorgenommen worden, stets wurden dabei auch Kontrollprobanden untersucht.

Bei 21 Studien konnten die Teilnehmer die Gerüche der getesteten Chemikalien deutlich wahrnehmen. Bei 19 dieser Studien berichteten die Probanden, die unter MCS litten, dabei vermehrt über typische Symptome.

Bei sieben Studien erfolgten die Provokationstests mit Konzentrationen, die unterhalb oder exakt an der Ge- ruchswahrnehmungsschwelle lagen. Bei sechs dieser Untersuchungen waren keine zuverlässigen Reaktionen bei den MCS-Patienten nachweisbar.

Bei drei Studien schließlich wurde während der Exposition die Wahrnehmung der auslösenden Stoffe durch simultane Exposition gegenüber anderen Gerüchen überdeckt. Bei keiner dieser Studien gaben die Probanden, die über MCS klagten, Symptome an, die der Provokation klar zuzuordnen waren.

Fazit: Probanden, die an MCS leiden, klagen offenbar nur dann über Symptome, wenn sie die auslösenden Substanzen deutlich riechen können. Die Tatsache, dass diese Reaktionen ausbleiben, wenn die Testpersonen den Geruch nicht wahrnehmen können, spricht für eine starke psychosomatische Komponente. af

Das-Munshi J et al. Multiple chemical sensitivities: A systematic review of provocation studies. J Allergy Clin Immunol 2006; 118: 1257-64 